\title{
Intravitreal bevacizumab injections versus dexamethasone implant for treatment-naïve retinal vein occlusion related macular edema
}

This article was published in the following Dove Press journal:

Clinical Ophthalmology

Number of times this article has been viewed

\author{
Ilkka Laine ${ }^{1-3}$ \\ Juha-Matti Lindholm ${ }^{1,2}$ \\ Petteri Ylinen ${ }^{1,4}$ \\ Raimo Tuuminen ${ }^{1,2,5}$ \\ 'Helsinki Retina Research Group, \\ University of Helsinki, Helsinki, ${ }^{2}$ Unit \\ of Ophthalmology, Kymenlaakso \\ Central Hospital, Kotka, Finland; \\ ${ }^{3}$ Department of Automation \\ and Electrical Engineering, Aalto \\ University, Helsinki, Finland; \\ ${ }^{4}$ Department of Ophthalmology, \\ Helsinki University Hospital, Helsinki, \\ Finland; ${ }^{5}$ The Insurance Centre, \\ Patient Insurance Centre, Helsinki, \\ Finland
}

\begin{abstract}
Purpose: To compare the short-term effects of three monthly intravitreal bevacizumab (IVB) injections to single dexamethasone (DEX) implantation in treatment-naïve patients with cystoid macular edema (CME) secondary to branch (BRVO) and central retinal vein occlusion (CRVO).
\end{abstract}

Design: A retrospective single-center study.

Subjects: A total of 135 eyes of 135 patients with BRVO $(n=83)$ and CRVO $(n=52)$.

Methods: Changes in clinical parameters were recorded before treatment and at the first and third month after commencement of IVB $(n=121)$ and DEX $(n=14)$.

Main outcome measures: Central retinal thickness (CRT), intraocular pressure (IOP), and best-corrected visual acuity (BCVA).

Results: The baseline parameters were comparable between IVB and DEX groups. After the first month, CRT decreased by $131.3 \pm 42.9 \mu \mathrm{m}$ in IVB and by $266.9 \pm 48.3 \mu \mathrm{m}$ in DEX (mean $\pm \mathrm{SEM} ; p=0.047$ ). IOP change was $-0.29 \pm 0.39 \mathrm{mmHg}$ in IVB and $+3.70 \pm 2.34 \mathrm{mmHg}$ in $\operatorname{DEX}(p=0.005)$. IOP elevation to $\geq 25 \mathrm{mmHg}$ and $\geq 5 \mathrm{mmHg}$ from the baseline was observed in two of the DEX- and in none of the IVB-treated eyes $(p=0.010)$. After the third month, no differences regarding CRT and IOP were observed between the treatment modalities. Moreover, BCVA gain was comparable between IVB $(0.37 \pm 0.05$ logarithm of minimum angle of resolution [logMAR] units $)$ and DEX (0.33 $\pm 0.30 \log$ MAR units $)$ groups.

Conclusion: DEX was associated with faster resolution of CME, but had greater probability for short-term IOP elevation when compared to IVB. After the third month, treatments were comparably effective. Anatomical outcomes and adverse drug reactions of IVB versus DEX should be considered case specifically in patients having CME secondary to BRVO/CRVO.

Keywords: anti-VEGF, bevacizumab, cystoid macular edema, dexamethasone implant, retinal vein occlusion

\section{Introduction}

Retinal vein occlusion (RVO) is the second most common retinal vascular disease after diabetic retinopathy. The prevalence of RVO in people over 40 years of age is $1 \%-2 \%$, and branch retinal vein occlusion (BRVO) is four times more common than central retinal vein occlusion (CRVO). ${ }^{1}$ RVO may result from mechanical damage in vascular wall or a local inflammatory process causing thrombosis, hypercoagulation, and stasis. One-third of the RVO cases develop to an ischemic form with worse prognosis. ${ }^{2,3}$

Disturbances in blood flow after RVO are associated with significant upregulation of vascular endothelial growth factor (VEGF) expression, vascular permeability and neovascularization, cystoid macular edema (CME), and loss of visual acuity (VA).
Correspondence: Raimo Tuuminen Unit of Ophthalmology, Kymenlaakso Central Hospital, Kotkantie 4I, 48210 Kotka, Finland

Tel +358504II 3870

Email raimo.tuuminen@helsinki.fi (c) (1) (5) 2017 Laine et al. This work is published and licensed by Dove Medical Press Limited. The full terms of this license are available at https://www.dovepress.com/terms.php cc. $\mathrm{BY}_{\mathrm{NC}}$ and incorporate the Creative Commons Attribution - Non Commercial (unported, v3.0) License (http://creativecommons.org/licenses/by-nc/3.0/). By accessing the work you hereby accept the Terms. Non-commercial uses of the work are permitted without any further permission from Dove Medical Press Limited, provided the work is properly attributed. For permission for commercial use of this work, please see paragraphs 4.2 and 5 of our Terms (https://www.dovepress.com/terms.php). 
Laser photocoagulation for macular edema in RVO, used to destroy the exuding blood vessels or to induce chorioretinal venous anastomosis, has at most marginal effect on improving VA. ${ }^{4,5}$ Numerous randomized clinical trials with antithrombotic therapy, fibrinolytic agents, anticoagulants, or with hemodilution have proved to be unsatisfactory in either results or because of their adverse effects. ${ }^{5}$

Intravitreally administered anti-VEGF agents are a wellestablished treatment for CME secondary to RVO, ${ }^{6-9}$ as well as intravitreal corticosteroids (triamcinolone acetonide and dexamethasone [DEX] implants). ${ }^{10,11}$ Besides partial VEGF inhibition, corticosteroids have an anti-inflammatory and blood-retinal barrier stabilizing effect on the retina by reducing the production of various inflammatory agents, such as tumor necrosis factor (TNF)- $\alpha$ and matrix metalloproteinases (MMPs), expression of intercellular adhesion molecule (ICAM)-1 on choroidal endothelial cells, and increasing the production of anti-inflammatory agents such as pigment epithelium-derived factor(PEDF). ${ }^{12,13}$ The choice of treatment modality between anti-VEGF agents and intravitreal corticosteroids may affect the patient's quality of life, workload of the treating clinic, total costs of care and the drug, and drug delivery-related adverse effects, most notably an increase in intraocular pressure (IOP), cataract progression, and endophthalmitis. ${ }^{14}$

The goal of this study was to compare the short-term treatment response and drug-related adverse effects of intravitreal bevacizumab (IVB) injections and DEX intravitreal implant in treatment-naïve BRVO and CRVO patients.

\section{Materials and methods Study design}

The study design was an institutional, retrospective, registerbased, observational study. Patients were admitted for the management of CME secondary to BRVO or CRVO in the Department of Ophthalmology, Kymenlaakso Central Hospital, Kotka, Finland. All patients included in the retrospective analysis were treatment-naïve regarding intravitreal medication. The diagnosis of BRVO/CRVO was carried out by a physician specialized in its diagnosis and treatment. The choice of therapies between three monthly anti-VEGF injections with bevacizumab (Avastin ${ }^{\circledR}$; Genentech, Inc., South San Francisco, CA, USA) or single DEX implant (Ozurdex ${ }^{\circledR}$; Allergan, Plc, Dublin, Ireland) was at the discretion of the treating physician. Main outcome measures were central retinal thickness (CRT), IOP, and best-corrected visual acuity (BCVA) at 1 and 3 months. The study was conducted as monitoring of clinical practice, and therefore informed patient consent was not required. The study was approved by the Institutional Review Board of the Research Director and
Chief Medical Officer of the Kymenlaakso Central Hospital. Confidentiality of the patient records was maintained when the clinical data were entered into a computer-based standardized data entry for analysis.

\section{Patients}

A total of 135 eyes of 135 treatment-naïve patients who were treated for CME secondary to BRVO/CRVO either with IVB injections or DEX implantation between January 1, 2011 and December 31, 2015, were included in the study. The inclusion criteria were: 1) CME from BRVO/CRVO and 2) follow-up of at least 3 months after commencement of the intravitreal therapy. Exclusion criteria were: ischemic BRVO/CRVO or less than three anti-VEGF injections given.

The patients were divided into two groups according to the type of the primary intravitreal treatment. Of the 135 study eyes, 121 were treated with three monthly bevacizumab injections and 14 were treated with single DEX implantation. The procedures were performed in the operation room by ophthalmologists or residents in ophthalmology. All patients were assessed before injections/implantations. VA, tonometry, and CRT derived from optical coherence tomography (OCT) imaging were recorded. Patients with DEX implantation were routinely followed by ophthalmologists at months 1 and 3. No postoperative endophthalmitis was observed in our study. The baseline variables are presented in Table 1.

\section{Clinical evaluation}

Clinical examination included bilateral VA testing at standardized light conditions, biomicroscopy, tonometry, and examination of the fundus. The classification for very low VA was on a semi-quantitative scale such as counting fingers (CF) and hand motion (HM). For statistical purposes, the Snellen values were transformed to the equivalent logarithm of minimum angle of resolution (logMAR) units. The very low VA measurements were converted as follows: CF 1.9, HM $2.3 \log$ MAR. None of the study patients had light perception or no light perception VA.

The mean CRT was recorded by spectral-domain OCT by an experienced ophthalmic nurse. At follow-up, 30-frame scans were performed with AutoRescan ${ }^{\mathrm{TM}}$ software and OCT analyses were compared to those done prior to intravitreal treatment (Heidelberg Eye Explorer version 1.9.10.0 and HRA/SPECTRALIS ${ }^{\circledR}$ Viewing Module version 6.0.9.0; Heidelberg Engineering GmbH, Heidelberg, Germany).

\section{Statistical analysis}

Data are given as mean \pm SEM and range (min-max) except the absolute number and proportion for nominal scale. 
Table I Baseline variables according to primary treatment for RVO-related CME

\begin{tabular}{|c|c|c|c|}
\hline Variables & IVB $(n=|2|)$ & DEX implant $(n=\mid 4)$ & $p$-value \\
\hline Gender (M/F) & $57: 64$ & $8: 6$ & 0.477 \\
\hline Age (years) & $72.8 \pm 1.06(45-94)$ & $72.1 \pm 3.88(47-92)$ & 0.849 \\
\hline RVO (branch:central) & $73: 48$ & $10: 4$ & 0.565 \\
\hline BCVA (logMAR) & $0.76 \pm 0.06(0-2.3)$ & $0.58 \pm 0.12(0-1.3)$ & 0.814 \\
\hline CRT $(\mu \mathrm{m})$ & $543.9 \pm 19.8(217-1,138)$ & $555.4 \pm 39.5(322-774)$ & 0.797 \\
\hline Lens status (phakic:IOL) & $87: 34$ & $10: 4$ & 1.000 \\
\hline Glaucoma medication (n/\%) & $15(12 \%)$ & $2(14 \%)$ & 0.690 \\
\hline IOP (mmHg) & $15.6 \pm 0.4(6-30)$ & $16.1 \pm 1.1(10-23)$ & 0.650 \\
\hline $\mathrm{IOP} \geq 22 \mathrm{mmHg}(\mathrm{n} / \%)$ & II (9\%) & I (7\%) & 1.000 \\
\hline $\mathrm{IOP} \geq 26 \mathrm{mmHg}(\mathrm{n} / \%)$ & $2(2 \%)$ & $0(0 \%)$ & 1.000 \\
\hline
\end{tabular}

Notes: Data are given as mean ( \pm SEM) and range or absolute number. For two-group comparisons, qualitative data was analyzed by two-factor $\chi^{2}$ test (or with the Fisher's exact test when values in any of the cells of a contingency table were below five) and continuous variables by Student's $t$-test.

Abbreviations: BCVA, best-corrected visual acuity; CRT, central retinal thickness; CME, cystic macular edema; DEX, dexamethasone; IOL, intraocular lens; IOP, intraocular pressure; IVB, intravitreal bevacizumab; RVO, retinal vein occlusion; SEM, standard error of the mean; MAR, minimum angle of resolution.

IBM SPSS Statistics 23 (SPSS Inc., Chicago, IL, USA) was used for statistical analyses. For two-group comparisons, qualitative data were analyzed by two-factor $\chi^{2}$ test (or with the Fisher's exact test when values in any of the cells of a contingency table were below five) and continuous variables by Student's $t$-test. A $p$-value $\leq 0.05$ was considered statistically significant.

\section{Results}

\section{Baseline patient and ophthalmic characteristics in bevacizumab and DEX treatment groups}

The gender distribution, mean age at the time of initiation of primary intravitreal treatment, type of RVO, BCVA, CRT, lens status, incidence of glaucoma, and IOP did not differ between the bevacizumab and DEX intravitreal implant treatment groups (Table 1).

\section{BCVA gain in short term is similar between the bevacizumab and DEX treatment groups}

The patients were treated either with three monthly injections of bevacizumab, the final follow-up was 1 month after the last injection in this group, or with single DEX implant, the follow-ups were at months 1 and 3 in this group. The BCVA gain in $\log$ MAR units was comparable between the treatment groups at these time points (Figure 1).

\section{DEX implantation results in faster resolution of CME compared to bevacizumab treatment group}

Next, we analyzed the effect of bevacizumab and DEX treatment on the mean CRT at months 1 and 3. At month 1, DEX treatment group resulted in greater CME resolution when compared to the bevacizumab treatment group ( $p=0.047$, Figure 2). However, at 3 months, the CRT change was comparable between the treatment groups (Figure 2).

\section{DEX implantation results in short-term but significant IOP increase compared to bevacizumab treatment group}

Finally, we analyzed the effect of bevacizumab and DEX treatment on IOP at months 1 and 3. At month 1, DEX treatment resulted in IOP increase when compared to the bevacizumab treatment group ( $p=0.005$, Figure 3 ). IOP $\geq 25 \mathrm{mmHg}$ and elevation $\geq 5 \mathrm{mmHg}$ from the baseline were observed more frequently in the DEX ( 2 of 14$)$ than bevacizumab ( 0 of 121$)$ treated eyes $(p<0.010$, data not shown). Topical IOP-lowering medication was temporarily prescribed for both patients (one with prostaglandin analogue and one

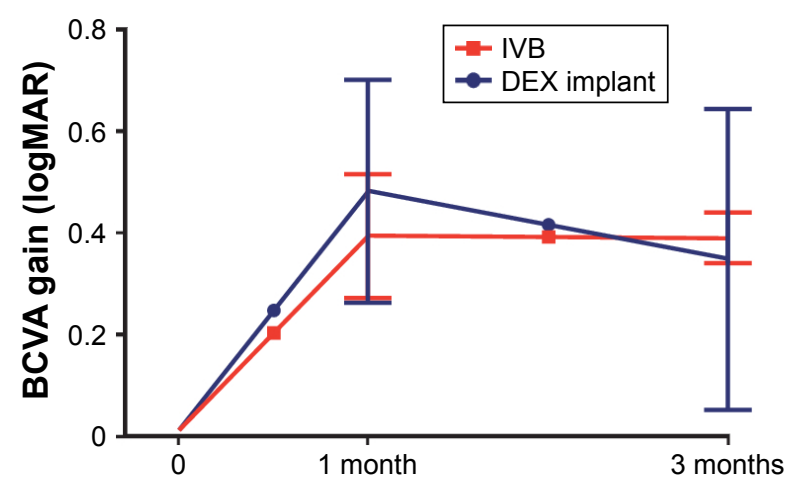

Figure I Three-month BCVA gain of the treatment-naive CME eyes secondary to retinal vein occlusion.

Notes: Patients were given either a) intravitreal bevacizumab (IVB) as three monthly injections with the final follow-up at month 3 or b) single DEX intravitreal implant with follow-ups at months I and 3. BCVA was recorded before injections/ implantation and at months I and 3; IVB ( $n=|2|)$, DEX implant $(n=14)$.

Abbreviations: BCVA, best-corrected visual acuity; CME, cystoid macular edema; MAR, minimum angle of resolution; DEX, dexamethasone. 


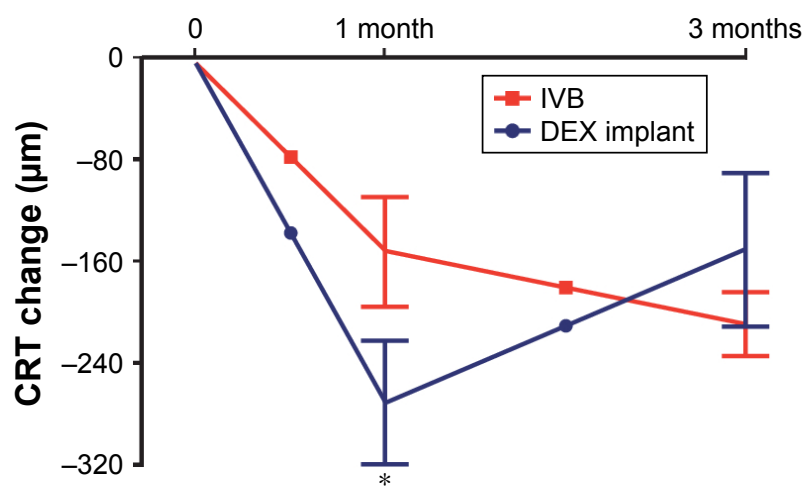

Figure 2 Three-month CRT decrease of the treatment-naive CME eyes secondary to retinal vein occlusion.

Notes: Patients were given either a) intravitreal bevacizumab (IVB) as three monthly injections with the final follow-up at month 3 or b) single DEX intravitreal implant with follow-ups at months I and 3. CRT was recorded before injections/ implantation and at months I and 3; IVB $(n=|2|)$, DEX implant $(n=\mid 4),{ }^{*} p<0.05$

Abbreviations: CRT, central retinal thickness; CME, cystoid macular edema; DEX, dexamethasone.

with combination of carbonic anhydrase inhibitor and beta blocker). At 3 months, the IOP levels did not significantly differ between the study groups (Figure 3).

\section{Discussion}

In RVO eyes, primary treatment with DEX implant resulted in more effective resolution of CME at month 1, but with a greater probability for IOP elevation when compared to IVB. At 3 months, these treatment modalities were equally effective regarding BCVA gain, CME resolution, and IOP change.

VEGF is a pivotal target to reduce CME in patients with RVO. In addition, various cytokines, vascular destabilizing and fibroproliferative factors such as angiopoietin (Ang)-2,

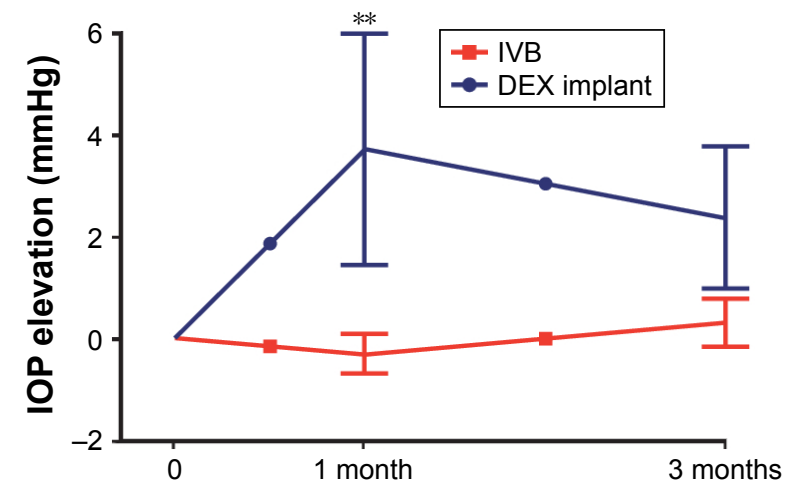

Figure 3 Three-month IOP change of the treatment-naïve CME eyes secondary to retinal vein occlusion.

Notes: Patients were given either a) intravitreal bevacizumab (IVB) as three monthly injections with the final follow-up at month 3 or b) single DEX intravitreal implant with follow-ups at months I and 3. IOP was recorded before injections/implantation and at months I and 3; IVB $(n=\mid 2 I)$, DEX implant $(n=\mid 4)$; ${ }^{* *} p<0.01$.

Abbreviations: IOP, intraocular pressure; CME, cystoid macular edema; DEX, dexamethasone. platelet derived growth factor (PDGF)-AA, transforming growth factor (TGF)- $\beta 1$, and matrix MMPs were reported to be elevated in aqueous and vitreous specimens of RVO eyes. ${ }^{15-18}$ Clinical experience has shown that some of the eyes with refractory CME are bad responders or resistant to anti-VEGF treatment. At the moment, lack of clinical parameters or reliable diagnostic tools to distinguish eyes with a prominent inflammatory component limit decision making in regard to primary treatment modality.

Administration of intravitreal triamcinolone acetonide or DEX implant reduced plural inflammatory cytokines in RVO eyes. ${ }^{19,20}$ Treatment with DEX implant has become an alternative for anti-VEGF agents, due to its long efficacy in CME resolution and visual improvement, ${ }^{11,21}$ especially when administered early after the onset of disease. ${ }^{22-24} \mathrm{DEX}$ implants have shown to be superior to anti-VEGF treatment regarding short-term CME resolution and VA recovery of treatment-naïve RVO patients. ${ }^{25,26}$ These findings are in accordance with our study, suggesting that short-term CME resolution is greater with DEX implant. The effect of DEX implant on CME resolution seemed to be waning by 3 months, particularly for CME resolution. This has been reported in previous studies. ${ }^{21,27}$

Retinal vascular disorders (including RVO) have direct impact on the costs of health care by increasing patient visits and medication and on other costs such as travel expenses and social services, eg, home care. They also cause indirect costs related to the lowered functional ability of the patient and the time and effort required by the treatment. DEX implant as a primary treatment for RVO is justified for patients in whom there is a reason to minimize the number of treatment and follow-up visits. On the other hand, the possible rise in IOP may increase the number of follow-up visits and precipitated development of cataract may lead to earlier cataract surgery. It has been reported before that combination treatment with both anti-VEGF and DEX implant may have stronger positive effect to either monotherapy, and thus could be a viable treatment option for some RVO patients. ${ }^{27}$

This study has several limitations. First, the patient selection may be biased, as the decision of primary treatment modality was nonrandomized and performed by the treating clinician. On the other hand, baseline patient and ophthalmic parameters did not significantly differ between the study groups. Second, longitudinal follow-up after the 3-month time-point was not systematic and thus not included in the analysis. Despite the limitations, our study emphasizes that primary treatment with IVB and single DEX implantation 
results in comparable BCVA gain. Anatomical outcomes and adverse drug reactions between intravitreal anti-VEGF and corticosteroid treatments should be case specifically considered in RVO patients.

\section{Acknowledgments}

The study was supported by grants from the Helsinki University Hospital Specific Catchment Area Clinical Research Grants, the Finnish Eye Foundation, Finnish Ophthalmological Society, the Nissi Foundation, and the Waldemar von Frenckell Foundation, Helsinki, Finland.

\section{Disclosure}

The authors report no conflicts of interest in this work.

\section{References}

1. Rogers S, McIntosh RL, Cheung N, et al. The prevalence of retinal vein occlusion: pooled data from population studies from the United States, Europe, Asia, and Australia. Ophthalmology. 2010;117(2): 313-319 e311.

2. Laatikainen L, Kohner EM. Fluorescein angiography and its prognostic significance in central retinal vein occlusion. $\mathrm{Br} J$ Ophthalmol. 1976;60(6):411-418.

3. McIntosh RL, Mohamed Q, Saw SM, Wong TY. Interventions for branch retinal vein occlusion: an evidence-based systematic review. Ophthalmology. 2007;114(5):835-854.

4. [No authors listed]. Evaluation of grid pattern photocoagulation for macular edema in central vein occlusion. The Central Vein Occlusion Study Group M report. Ophthalmology. 1995;102(10):1425-1433.

5. Mohamed Q, McIntosh RL, Saw SM, Wong TY. Interventions for central retinal vein occlusion: an evidence-based systematic review. Ophthalmology. 2007;114(3):507-519, 524.

6. Brown DM, Campochiaro PA, Singh RP, et al. Ranibizumab for macular edema following central retinal vein occlusion: six-month primary end point results of a phase III study. Ophthalmology. 2010;117(6):1124-1133.e1121.

7. Kinge B, Stordahl PB, Forsaa V, et al. Efficacy of ranibizumab in patients with macular edema secondary to central retinal vein occlusion: results from the sham-controlled ROCC study. Am J Ophthalmol. 2010;150(3):310-314.

8. Epstein DL, Algvere PV, von Wendt G, Seregard S, Kvanta A. Bevacizumab for macular edema in central retinal vein occlusion: a prospective, randomized, double-masked clinical study. Ophthalmology. 2012; 119(6):1184-1189.

9. Tan MH, McAllister IL, Gillies ME, et al. Randomized controlled trial of intravitreal ranibizumab versus standard grid laser for macular edema following branch retinal vein occlusion. Am J Ophthalmol. 2014; 157(1):237-247 e231.

10. Ip MS, Scott IU, VanVeldhuisen PC, et al. A randomized trial comparing the efficacy and safety of intravitreal triamcinolone with observation to treat vision loss associated with macular edema secondary to central retinal vein occlusion: the Standard Care vs Corticosteroid for Retinal Vein Occlusion (SCORE) study report 5. Arch Ophthalmol. 2009;127(9):1101-1114
11. Haller JA, Bandello F, Belfort R Jr, et al. Randomized, sham-controlled trial of dexamethasone intravitreal implant in patients with macular edema due to retinal vein occlusion. Ophthalmology. 2010;117(6): 1134-1146.e1133.

12. Floman N, Zor U. Mechanism of steroid action in ocular inflammation: inhibition of prostaglandin production. Invest Ophthalmol Vis Sci. 1977;16(1):69-73.

13. Wang K, Wang Y, Gao L, Li X, Li M, Guo J. Dexamethasone inhibits leukocyte accumulation and vascular permeability in retina of streptozotocin-induced diabetic rats via reducing vascular endothelial growth factor and intercellular adhesion molecule-1 expression. Biol Pharm Bull. 2008;31(8):1541-1546.

14. Garweg JG, Zandi S. Retinal vein occlusion and the use of a dexamethasone intravitreal implant (Ozurdex(R)) in its treatment. Graefes Arch Clin Exp Ophthalmol. 2016;254(7):1257-1265.

15. Tuuminen R, Loukovaara S. Increased intravitreal angiopoietin-2 levels in patients with retinal vein occlusion. Acta Ophthalmol. 2014;92(2):e164-e165.

16. Jung SH, Kim KA, Sohn SW, Yang SJ. Association of aqueous humor cytokines with the development of retinal ischemia and recurrent macular edema in retinal vein occlusion. Invest Ophthalmol Vis Sci. 2014;55(4):2290-2296.

17. Tuuminen R, Loukovaara S. High intravitreal TGF-beta1 and MMP-9 levels in eyes with retinal vein occlusion. Eye. 2014;28(9):1095-1099.

18. Ehlken C, Grundel B, Michels D, et al. Increased expression of angiogenic and inflammatory proteins in the vitreous of patients with ischemic central retinal vein occlusion. PLoS One. 2015;10(5):e0126859.

19. Sohn HJ, Han DH, Lee DY, Nam DH. Changes in aqueous cytokines after intravitreal triamcinolone versus bevacizumab for macular oedema in branch retinal vein occlusion. Acta Ophthalmol. 2014; 92(3):e217-e224.

20. Rezar-Dreindl S, Eibenberger K, Pollreisz A, et al. Effect of intravitreal dexamethasone implant on intra-ocular cytokines and chemokines in eyes with retinal vein occlusion. Acta Ophthalmol. 2017;95(2):e119-e127.

21. Bezatis A, Spital G, Hohn F, et al. Functional and anatomical results after a single intravitreal Ozurdex injection in retinal vein occlusion: a 6-month follow-up - the SOLO study. Acta Ophthalmol. 2013;91(5): e340-e347.

22. Yeh WS, Haller JA, Lanzetta P, et al. Effect of the duration of macular edema on clinical outcomes in retinal vein occlusion treated with dexamethasone intravitreal implant. Ophthalmology. 2012;119(6):1190-1198.

23. Mayer WJ, Remy M, Wolf A, et al. Comparison of intravitreal bevacizumab upload followed by a dexamethasone implant versus dexamethasone implant monotherapy for retinal vein occlusion with macular edema. Ophthalmologica. 2012;228(2):110-116.

24. Eter N, Mohr A, Wachtlin J, et al. Dexamethasone intravitreal implant in retinal vein occlusion: real-life data from a prospective, multicenter clinical trial. Graefes Arch Clin Exp Ophthalmol. 2017;255(1):89.

25. Chiquet $\mathrm{C}$, Dupuy $\mathrm{C}$, Bron AM, et al. Intravitreal dexamethasone implant versus anti-VEGF injection for treatment-naive patients with retinal vein occlusion and macular edema: a 12-month follow-up study. Graefes Arch Clin Exp Ophthalmol. 2015;253(12):2095-2102.

26. Yumusak E, Buyuktortop N, Ornek K. Early results of dexamethasone implant, ranibizumab, and triamcinolone in macular edema due to branch retinal vein occlusion. Eur J Ophthalmol. 2016;26(1):54-59.

27. Mayer WJ, Wolf A, Kernt M, et al. Twelve-month experience with ozurdex for the treatment of macular edema associated with retinal vein occlusion. Eye. 2013;27(7):816-822. 


\section{Publish your work in this journal}

Clinical Ophthalmology is an international, peer-reviewed journal covering all subspecialties within ophthalmology. Key topics include: Optometry; Visual science; Pharmacology and drug therapy in eye diseases; Basic Sciences; Primary and Secondary eye care; Patient Safety and Quality of Care Improvements. This journal is indexed on

Submit your manuscript here: http://www.dovepress.com/clinical-ophthalmology-journal
PubMed Central and CAS, and is the official journal of The Society of Clinical Ophthalmology (SCO). The manuscript management system is completely online and includes a very quick and fair peer-review system, which is all easy to use. Visit http://www.dovepress.com/ testimonials.php to read real quotes from published authors. 